\title{
The prevalence and characteristics of adults with latent tuberculous infection in the United States and the implications for healthcare in Texas
}

\author{
Shazma Khan BSA, Crystal Ike BS, Jeff Dennis PhD, Kenneth Nugent MD
}

\begin{abstract}
The Centers for Disease Control and Prevention reported 8,916 cases of tuberculosis in 2019. Reducing the number of cases of active tuberculosis requires identification of patients with latent tuberculous infections (LTBI). Optimal screening for $L T B I$ requires information about the demographics and characteristics of people who are more likely to have had tuberculous infection. Information from the 2011-2012 National Health and Nutrition Examination Survey (NHANES) was used to determine the number and characteristics of adults from a representative sample of the United States who had LTBI. Latent tuberculous infection was identified either by a positive skin test or by a positive QuantiFERON blood test. Information about the number of patients with active tuberculosis in Texas was determined from reports from the Texas State Department of Health Services. The NHANES database for the years 2011-2012 included 5,684 adults. Participants with a positive QuantiFERON blood test were more likely in the age group 45-64, male, foreign born, and have less than a high school education. Participants with a positive skin test had similar characteristics. Participants who had both a positive skin test and positive QuantiFERON test were more likely to be in the age group 45-64, males, foreign born, and Hispanic. In addition, they had diabetes, self-reported fair/poor health, and an educational level less than high school. In the State of Texas tuberculosis occurred more frequently in individuals older than 75 who were male and were not US born Texas residents. Important clinical diagnoses included diabetes, alcohol abuse, correctional facility residence, non-injection drug use, positive HIV status, and homelessness. Information from the NHANES study and from the State Department of Health Services in Texas provides information needed to develop screening programs for latent tuberculosis and active tuberculosis.
\end{abstract}

Keywords: Latent tuberculous infection, skin test, QuantiFERON test, risk factors, NHANES

\section{INTRODUCTION}

The Centers for Disease Control and Prevention (CDC) provides information about the number of cases of active tuberculosis each year in the United States and the case rate per 100,000 population. ${ }^{1}$ The

Corresponding author: Kenneth Nugent Contact Information: Kenneth.Nugent@ttuhsc.edu DOI: $10.12746 /$ swrccc.v9i39.847 demographic characteristics of patients with active tuberculosis provide a reasonable description of people in United States who likely have latent tuberculous infection (LTBI). However, the CDC does not routinely do population-based studies to determine the prevalence of LTBI. This information is potentially available from local and state departments of health, such as the Texas Department of State Health Services, but information from these organizations largely reflects contact investigation of active cases. The National Health and Nutrition Examination Survey (NHANES) project does determine the prevalence of LTBI periodically, 
and this provides the best overall estimate of LTBI in non-institutionalized adults in the United States. ${ }^{2,3}$

This report summarizes information in the 2012 NHANES survey which used both skin testing and QuantiFERON testing to determine the prevalence of LTBI in a representative sample of adults in United States. The demographic characteristics of individuals with LTBI in the NHANES database were compared to demographic characteristics of patients with active TB infection collected by State Department of Health in Texas. ${ }^{4}$ This information can help healthcare workers identify patients at a higher risk for LTBI who might warrant testing.

\section{Methods}

This study used the 2011-2012 National Health and Nutrition Examination Survey, a nationally representative cross-sectional sample of U.S. residents collected biennially. ${ }^{2}$ This survey data collection includes an extensive health questionnaire, a physical examination, and laboratory tests. The NHANES 2011-2012 data collection was the most recent data release in which tuberculosis testing was performed on participants. The population of interest was identified using two different tests. A purified protein derivative (PPD) skin test was positive if the skin test reaction $>10 \mathrm{~mm}$, as measured during the NHANES examination by trained staff. A second test identified positive tuberculous infection using a Quantiferon-TB Gold in tube blood test. For comparison purposes, four groups were created, including 1) no positive TB test, 2) positive PPD test only, 3) positive Quantiferon test only, and 4) both positive PPD and Quantiferon test.

NHANES also collects data on prescription medication information from respondents to determine which medications they have been prescribed in the past 30 days. From these data, the use of isoniazid, rifampin, ethambutol, and pyrazinamide was identified to determine treatment of tuberculosis with medication. Analysis was conducted using Stata 16.1 with population weights via the "svy" command to account for complex survey design (StataCorp, 2019). Case counts below frequently do not match up to percentages because of weighting for population representativeness. Mean and proportions were tested using Wald and chi-square tests, respectively.

Information was also collected from the Texas TB Surveillance Report for 2017 prepared in August $2018 .{ }^{4}$

\section{REsults}

There were 5,864 adult survey participants in the 2011-2012 NHANES database. Of these participants, $5,232(89.2 \%)$ had blood drawn for the QuantiFERON test, $4,491(76.6 \%)$ had a PPD test placed and measured, and 4,441 (75.7\%) had both tests.

Of the 5,232 participants who had blood drawn for a QuantiFERON test, 519 had a positive result indicative of LTBI (Table 1). Using population weights, the prevalence of a positive QuantiFERON test was 5.9\%. The prevalence of LTBI was highest in those aged $65+$ $(162,8.8 \%)$. Male participants had a higher prevalence of infection $(299,6.9 \%)$ than female participants $(220$, $4.9 \%)$. Foreign-born participants had a significantly higher prevalence $(339,17.0 \%)$ than U.S.-born persons (180, 3.4\%). Non-Hispanic whites had the lowest prevalence of LTBI (74, 3.2\%). A higher prevalence of LTBI was found in Hispanics (168, 12.4\%), non-Hispanic blacks $(125,7.6 \%)$, and other races $(152,14.0 \%)$. The prevalence of LTBI was increased in those who had less than a high school education (191, 11.3\%), compared to those with a high school diploma $(105,7.1 \%)$ or some college education (99, 3.5\%).

Among the 4,491 participants who had a PPD test placed and measured, 393 (4.6\%, weighted) had a positive result indicative of LTBI (Table 2). The characteristics of those who tested positive on the PPD skin test were similar to those with a positive QuantiFERON test. The highest prevalence was in those aged 45-64 (172, 5.3\%). There was, however, a lower LTBI prevalence in those in the $65+$ age group $(80,3.7 \%)$ when compared with results on the QuantiFERON test (162, $8.8 \%$ ). In addition, LTBI was more frequent in foreign-born participants $(284,19.3 \%)$ than the U.S-born participants $(109,1.7 \%)$. The PPD positive prevalence also followed similar patterns as QuantiFERON positive prevalence in racial and ethnic groups and in the level of education (Table 1). 
Table 1. Quantiferon Test Results in NHANES 2011-2012

\begin{tabular}{|c|c|c|c|c|}
\hline & $\begin{array}{c}\text { Negative } \\
\text { Blood Test }\end{array}$ & $\begin{array}{c}\text { Positive } \\
\text { Blood Test }\end{array}$ & Chi-Square & P-Value \\
\hline \multicolumn{5}{|l|}{ Age Groups, Years } \\
\hline $18-44$ & $2,328(48.59)$ & $125(31.20)$ & 37.8974 & $<0.001$ \\
\hline $45-64$ & $1,474(34.94)$ & $232(43.27)$ & & \\
\hline $65+$ & $911(16.47)$ & $162(25.53)$ & & \\
\hline \multicolumn{5}{|l|}{ Sex } \\
\hline Female & $2,427(52.35)$ & $220(43.72)$ & 8.6004 & 0.004 \\
\hline Male & $2,286(47.65)$ & $299(56.28)$ & & \\
\hline \multicolumn{5}{|l|}{ Nativity } \\
\hline U.S.-born & $3,463(84.27)$ & $180(48.14)$ & 441.0606 & $<0.001$ \\
\hline Foreign-born & $1,247(15.73)$ & $339(51.86)$ & & \\
\hline \multicolumn{5}{|l|}{ Race/Ethnicity } \\
\hline NH white & $1,841(68.36)$ & $74(36.10)$ & 156.4733 & $<0.001$ \\
\hline NH black & $1,239(11.06)$ & $125(14.62)$ & & \\
\hline Hispanic & $910(13.57)$ & $168(30.91)$ & & \\
\hline Other & $723(7.01)$ & $152(18.37)$ & & \\
\hline \multicolumn{5}{|l|}{ PPD } \\
\hline Induration $\leq 10 \mathrm{~mm}$ & $3,842(97.37)$ & $210(61.00)$ & 689.545 & $<0.001$ \\
\hline$>10 \mathrm{~mm}$ & $185(2.63)$ & $204(39.00)$ & & \\
\hline \multicolumn{5}{|l|}{ BMI, $\mathrm{kg} / \mathrm{m}^{2}$} \\
\hline$<18.5$ & $104(1.93)$ & $12(2.27)$ & 1.3291 & 0.6897 \\
\hline 18.5 to $<25$ & $1,431(29.90)$ & $165(32.62)$ & & \\
\hline 25 to $<30$ & $1,463(33.76)$ & $173(31.57)$ & & \\
\hline $30+$ & $1,644(34.40)$ & $165(33.55)$ & & \\
\hline \multicolumn{5}{|l|}{ Self-Reported Health } \\
\hline Fair/poor & $1,007(15.87)$ & $153(25.20)$ & 25.3361 & $<0.001$ \\
\hline Good & $1,754(35.58)$ & $208(38.67)$ & & \\
\hline Very good/excellent & $1,951(48.54)$ & $158(36.14)$ & & \\
\hline \multicolumn{5}{|l|}{ Education } \\
\hline$<\mathrm{HS}$ & $947(15.32)$ & $191(30.81)$ & 63.1936 & $<0.001$ \\
\hline HS diploma & 935 (19.93) & $105(23.98)$ & & \\
\hline Some college & $1,412(33.01)$ & $99(18.75)$ & & \\
\hline College or more & $1,149(31.73)$ & $118(26.46)$ & & \\
\hline
\end{tabular}


Table 2. Purified Protein Derivative Skin Test Results in NHANES 2011-2012

\begin{tabular}{|c|c|c|c|c|}
\hline & $\leq 10 \mathrm{~mm}$ & $>10 \mathrm{~mm}$ & Chi-Square & P-Value \\
\hline \multicolumn{5}{|l|}{ Age Groups, Years } \\
\hline $18-44$ & $1,939(46.67)$ & $141(44.13)$ & 3.8522 & 0.1859 \\
\hline $45-64$ & $1,319(36.23)$ & $172(42.39)$ & & \\
\hline $65+$ & $840(17.10)$ & $80(13.48)$ & & \\
\hline \multicolumn{5}{|l|}{ Sex } \\
\hline Female & $2,097(51.98)$ & $173(47.19)$ & 1.8105 & 0.1146 \\
\hline Male & $2,001(48.02)$ & $220(52.81)$ & & \\
\hline \multicolumn{5}{|l|}{ Nativity } \\
\hline U.S.-born & $3,108(85.85)$ & $109(30.01)$ & 441.0606 & $<0.001$ \\
\hline Foreign-born & $987(14.15)$ & $284(69.99)$ & & \\
\hline \multicolumn{5}{|l|}{ Race/Ethnicity } \\
\hline NH white & $1,658(69.46)$ & $23(15.07)$ & 302.4331 & $<0.001$ \\
\hline NH black & $1,092(11.08)$ & $105(17.86)$ & & \\
\hline Hispanic & $802(13.36)$ & $145(44.42)$ & & \\
\hline Other & $546(6.09)$ & $120(22.66)$ & & \\
\hline \multicolumn{5}{|l|}{ TB Blood Test } \\
\hline Negative & $3,842(96.49)$ & $185(53.79)$ & 689.5446 & $<0.001$ \\
\hline Positive & $210(3.51)$ & $204(46.21)$ & & \\
\hline \multicolumn{5}{|l|}{ BMI, $\mathrm{kg} / \mathrm{m}^{2}$} \\
\hline$<18.5$ & $88(1.88)$ & $10(1.87)$ & 0.4151 & 0.909 \\
\hline 18.5 to $<25$ & $1,192(28.83)$ & $126(30.24)$ & & \\
\hline 25 to $<30$ & $1,289(33.79)$ & $134(34.51)$ & & \\
\hline $30+$ & $1,471(35.50)$ & $123(33.38)$ & & \\
\hline \multicolumn{5}{|l|}{ Self-Reported Health } \\
\hline Fair/poor & $921(16.49)$ & $101(25.91)$ & 20.1842 & $<0.001$ \\
\hline Good & $1,519(35.99)$ & $171(40.84)$ & & \\
\hline Very good/excellent & $1,658(47.52)$ & $121(33.25)$ & & \\
\hline \multicolumn{5}{|l|}{ Education } \\
\hline$<\mathrm{HS}$ & $858(15.52)$ & $143(38.48)$ & 73.1826 & $<0.001$ \\
\hline HS diploma & $844(20.84)$ & $70(17.63)$ & & \\
\hline Some college & $1,228(33.10)$ & $84(22.23)$ & & \\
\hline College or more & $945(30.55)$ & $87(21.67)$ & & \\
\hline
\end{tabular}


Table 3. Clinical Parameters and Latent TB Test Results in NHANES 2011-2012

\begin{tabular}{|c|c|c|c|c|}
\hline & $\begin{array}{c}\text { Negative } \\
\text { Quantiferon }\end{array}$ & $\begin{array}{c}\text { Positive } \\
\text { Quantiferon }\end{array}$ & $\mathbf{F}$ & P-Value \\
\hline Hemoglobin A1c, \%* & $5.62(0.91)$ & $5.89(1.45)$ & 15.03 & 0.0012 \\
\hline Fasting glucose, $\mathrm{mg} / \mathrm{dL}$ & $104.32(27.99)$ & $109.01(39.99)$ & 2.63 & 0.1235 \\
\hline Albumin, $\mathrm{g} / \mathrm{dL}$ & $4.32(0.33)$ & $4.26(0.43)$ & 7.45 & 0.0143 \\
\hline Creatinine, $\mathrm{mg} / \mathrm{dL}$ & $0.88(0.34)$ & $0.87(0.36)$ & 0.75 & 0.3977 \\
\hline GFR, $\mathrm{ml} / \mathrm{min}$ & $90.09(24.75)$ & $92.40(34.55)$ & 2.57 & 0.1276 \\
\hline \multicolumn{5}{|l|}{ GFR Categories } \\
\hline$<60$ & $416(8.26)$ & $49(8.76)$ & 1.7072 & 0.3334 \\
\hline 60 to $<90$ & $1,795(44.81)$ & $204(40.93)$ & & \\
\hline \multirow[t]{2}{*}{$90+$} & $2,382(46.93)$ & $252(50.32)$ & & \\
\hline & $P P D \leq 10 \mathrm{~mm}$ & PPD $>10 \mathrm{~mm}$ & $\mathbf{F}$ & P-Value \\
\hline Hemoglobin A1c, \% & $5.64(0.91)$ & $5.84(1.58)$ & 7.34 & 0.0149 \\
\hline Fasting glucose, $\mathrm{mg} / \mathrm{dL}$ & $104.50(28.28)$ & $110.70(49.76)$ & 3.15 & 0.0936 \\
\hline Albumin, $\mathrm{g} / \mathrm{dL}$ & $4.31(0.33)$ & $4.31(0.44)$ & 0.01 & 0.9437 \\
\hline Creatinine, mg/dL & $0.88(0.34)$ & $0.85(0.41)$ & 4.7 & 0.0446 \\
\hline GFR, $\mathrm{ml} / \mathrm{min}$ & $89.72(24.98)$ & $96.70(35.69)$ & 9.27 & 0.0073 \\
\hline \multicolumn{5}{|l|}{ GFR Categories } \\
\hline$<60$ & $378(8.66)$ & $22(3.04)$ & 13.6076 & 0.0037 \\
\hline 60 to $<90$ & $1,574(45.59)$ & $159(40.13)$ & & \\
\hline $90+$ & $2,049(45.75)$ & $203(56.82)$ & & \\
\hline
\end{tabular}

* Numbers are reported as mean and standard deviation.

There were no important clinical differences in routine lab tests which might reflect chronic disease between participants with a positive test and participants with a negative test (Table 3 ).

Table 4 includes comparisons of NHANES participants who had a negative QuantiFERON test and a negative skin test with participants who had a positive QuantiFERON test, or a positive skin test, or both a positive skin test and QuantiFERON test. Subjects who had both test positives were more likely to be in the age group 45-64, males, foreign-born, and Hispanic, and have diabetes, self-reported fair/ poor health, and an educational level less than high school. There were no clinically important differences among these 4 categories in hemoglobin A1c, fasting glucose, albumin, creatinine, and estimated GFRs.

Analysis of use of antituberculous medication identified fewer than 10 individuals currently taking these medications. NHANES is not designed for population estimates using subsamples less than 30 , therefore, we do not report exact numbers in this section because the data do not support any analysis. Regardless, the lack of active anti-tuberculous medication use suggests extremely low use in this population.

The Texas TB Surveillance Report from 2017 was used to derive data on the characteristics of patients with active tuberculosis in all Texas counties 
Table 4. Comparison of Skin Test and QuantiFERON Results in NHANES 2011-2012

\begin{tabular}{|c|c|c|c|c|c|c|}
\hline & No Positive & $\begin{array}{c}\text { QFT-Only } \\
\text { Positive }\end{array}$ & $\begin{array}{l}\text { PPD-Only } \\
\text { Positive }\end{array}$ & $\begin{array}{c}\text { PPD \& QFT } \\
\text { Positive }\end{array}$ & $\begin{array}{c}\text { Chi } \\
\text { Square }\end{array}$ & P Value \\
\hline \multicolumn{7}{|l|}{ Age Groups, Years } \\
\hline $18-44^{*}$ & $1,867(47.50)$ & $55(32.05)$ & $96(54.54)$ & $44(32.10)$ & 34.63 & 0.0003 \\
\hline $45-64$ & $1,215(35.70)$ & $84(40.61)$ & $67(38.74)$ & $104(47.00)$ & & \\
\hline $65+$ & $760(16.80)$ & $71(27.33)$ & $22(6.72)$ & $56(20.91)$ & & \\
\hline \multicolumn{7}{|l|}{ Sex } \\
\hline Female & $1,998(52.61)$ & $81(40.49)$ & $84(47.44)$ & $87(46.64)$ & 10.51 & 0.0071 \\
\hline Male & $1,844(47.39)$ & $129(59.51)$ & $101(52.56)$ & $117(53.36)$ & & \\
\hline \multicolumn{7}{|l|}{ Nativity } \\
\hline U.S.-born & $2,983(86.68)$ & $87(59.57)$ & $47(32.10)$ & $60(27.37)$ & 511.98 & $<0.001$ \\
\hline Foreign-born & $857(13.32)$ & $123(40.43)$ & $138(67.90)$ & $144(72.63)$ & & \\
\hline \multicolumn{7}{|l|}{ Race/Ethnicity } \\
\hline NH white & $1,589(70.01)$ & $51(51.18)$ & $14(19.49)$ & $9(10.11)$ & 338.88 & $<0.001$ \\
\hline NH black & $1,041(11.18)$ & $38(9.23)$ & $45(15.03)$ & $57(20.58)$ & & \\
\hline Hispanic & $724(12.95)$ & $68(26.35)$ & $65(42.90)$ & $79(46.27)$ & & \\
\hline Other & $488(5.86)$ & $53(13.25)$ & $61(22.58)$ & $59(23.04)$ & & \\
\hline \multicolumn{7}{|l|}{ BMI, $\mathrm{kg} / \mathrm{m}^{2}$} \\
\hline$<18.5$ & $81(1.84)$ & $5(2.89)$ & $5(1.82)$ & $5(1.96)$ & 3.85 & 0.8494 \\
\hline 18.5 to $<25$ & $1,112(28.69)$ & $67(33.91)$ & $62(29.70)$ & $64(31.26)$ & & \\
\hline 25 to $<30$ & $1,207(33.99)$ & $71(29.24)$ & $63(35.80)$ & $67(32.15)$ & & \\
\hline $30+$ & $1,388(35.48)$ & $65(33.97)$ & $55(32.69)$ & $68(34.62)$ & & \\
\hline \multicolumn{7}{|c|}{ Ever Told You Have Diabetes } \\
\hline No & $3,403(91.41)$ & $163(81.49)$ & $166(93.03)$ & $173(86.59)$ & 20.10 & 0.0016 \\
\hline Yes & $439(8.59)$ & $47(18.51)$ & $19(6.97)$ & $31(13.41)$ & & \\
\hline \multicolumn{7}{|l|}{ Self-Reported Health } \\
\hline Fair/poor & $844(16.20)$ & $67(24.08)$ & $47(24.95)$ & $53(26.88)$ & 29.47 & $<0.001$ \\
\hline Good & $1,422(35.85)$ & $80(37.59)$ & $75(38.36)$ & $95(43.89)$ & & \\
\hline Very good-excellent & $1,576(47.94)$ & $63(38.33)$ & $63(36.70)$ & $56(29.24)$ & & \\
\hline \multicolumn{7}{|l|}{ Education } \\
\hline$<\mathrm{HS}$ & $764(14.98)$ & $80(27.42)$ & $61(37.75)$ & $79(38.82)$ & 109.77 & $<0.001$ \\
\hline HS diploma & $779(20.42)$ & $56(32.28)$ & $36(16.72)$ & $34(18.83)$ & & \\
\hline Some college & $1,184(33.69)$ & $33(16.73)$ & $44(23.74)$ & $40(20.66)$ & & \\
\hline College or more & $896(30.91)$ & $40(23.58)$ & $39(21.79)$ & $48(21.69)$ & & \\
\hline
\end{tabular}

* Numbers are reported as number and percent. 
Table 5. Tuberculosis Cases in Texas

\begin{tabular}{|c|c|c|c|c|c|c|c|c|c|c|}
\hline & \multicolumn{2}{|c|}{2013} & \multicolumn{2}{|r|}{2014} & \multicolumn{2}{|r|}{2015} & \multicolumn{2}{|c|}{2016} & \multicolumn{2}{|c|}{2017} \\
\hline & Count & Prevalence & Count & Prevalence & Count & Prevalence & Count & Prevalence & Count & Prevalence \\
\hline \multicolumn{11}{|c|}{ Age Groups, Years } \\
\hline $0-5$ & 55 & $1 \%$ & 52 & $4 \%$ & 49 & $4 \%$ & 48 & $4 \%$ & 38 & $3 \%$ \\
\hline $6-13$ & 15 & $2 \%$ & 20 & $2 \%$ & 27 & $2 \%$ & 19 & $2 \%$ & 16 & $1 \%$ \\
\hline $14-17$ & 28 & $11 \%$ & 25 & $2 \%$ & 31 & $2 \%$ & 29 & $2 \%$ & 28 & $2 \%$ \\
\hline $18-24$ & 129 & $17 \%$ & 116 & $9 \%$ & 112 & $8 \%$ & 130 & $10 \%$ & 84 & $7 \%$ \\
\hline $25-34$ & 202 & $13 \%$ & 213 & $17 \%$ & 231 & $17 \%$ & 204 & $16 \%$ & 173 & $15 \%$ \\
\hline $35-44$ & 163 & $18 \%$ & 201 & $16 \%$ & 211 & $16 \%$ & 192 & $15 \%$ & 196 & $17 \%$ \\
\hline $45-54$ & 223 & $16 \%$ & 213 & $17 \%$ & 220 & $17 \%$ & 218 & $17 \%$ & 179 & $16 \%$ \\
\hline $55-64$ & 198 & $9 \%$ & 216 & $17 \%$ & 225 & $17 \%$ & 194 & $16 \%$ & 187 & $17 \%$ \\
\hline $65-74$ & 106 & $8 \%$ & 117 & $9 \%$ & 119 & $9 \%$ & 118 & $9 \%$ & 118 & $10 \%$ \\
\hline $75+$ & 102 & $0 \%$ & 96 & $8 \%$ & 108 & $8 \%$ & 98 & $8 \%$ & 108 & $10 \%$ \\
\hline \multicolumn{11}{|l|}{ Sex } \\
\hline Male & 798 & $65 \%$ & 840 & $66 \%$ & 857 & $64 \%$ & 819 & $66 \%$ & 734 & $65 \%$ \\
\hline Female & 423 & $35 \%$ & 428 & $34 \%$ & 476 & $36 \%$ & 427 & $34 \%$ & 393 & $35 \%$ \\
\hline \multicolumn{11}{|c|}{ US-Born Race/Ethnicity } \\
\hline White & 173 & $14 \%$ & 123 & $10 \%$ & 143 & $11 \%$ & 110 & $9 \%$ & 96 & $9 \%$ \\
\hline Black & 229 & $19 \%$ & 229 & $18 \%$ & 250 & $19 \%$ & 235 & $19 \%$ & 213 & $19 \%$ \\
\hline Hispanic & 623 & $51 \%$ & 690 & $54 \%$ & 671 & $50 \%$ & 666 & $53 \%$ & 593 & $53 \%$ \\
\hline API & 196 & $16 \%$ & 231 & $18 \%$ & 266 & $20 \%$ & 238 & $19 \%$ & 295 & $26 \%$ \\
\hline AIAN & 0 & $0 \%$ & 3 & $0 \%$ & 3 & $0 \%$ & 1 & $0 \%$ & 0 & $0 \%$ \\
\hline $\begin{array}{c}\text { Non-US } \\
\text { Born }\end{array}$ & 674 & $55 \%$ & 766 & $60 \%$ & 772 & $58 \%$ & 739 & $59 \%$ & 689 & $61 \%$ \\
\hline
\end{tabular}

(Table 5). There were 6,200 active TB cases in the years 2013-2017. The most recent 2017 data show that individuals older than 75 had the highest rate of infection at 7.8 per 100,000 Texas residents, followed by those aged $55-64$ (5.9 per 100,000), those aged 65-74 (5.6 per 100,000), and then those aged 45-54 $(5.0$ per 100,000$)$. Infection rates were higher in males (5.2 per 100,000) than females (2.8 per 100, 000), and more prevalent in non-U.S.-born Texas residents ( $61 \%$ of cohort). Among the U.S.-born, the highest proportion of TB cases was reported in Hispanics $(46 \%)$, followed by Blacks (30\%). Other important risk factors among TB cases included diabetes with 19\% of positive TB cases in 2017, alcohol abuse (11\%), correctional facility residence $(8 \%)$, non-injection drug use $(8 \%)$, positive HIV status $(6 \%)$, and homelessness (4\%).

\section{Discussion}

Approximately $8 \%$ of the adults who participated in the 2011-2012 NHANES survey had a positive QuantiFERON or PPD test. Based on the US population in 2012 of 240 million adults, this means that approximately 19 million adults have latent tuberculosis; in Texas the number of cases is approximately 
2.6 million. Positive tests were more frequent in males, US born Hispanics, and adults with less than high school education. These individuals had core laboratory tests similar to participants who had negative tests. Mancuso et al. analyzed the 2011-2012 NHANES and determined that there was very little change in the prevalence of latent tuberculosis between the years 2000 and 2011 in the United States. ${ }^{3}$ Haddad and coauthors have estimated the number of LTBI in the United States by using the number of active disease cases. ${ }^{5}$ They estimated that progression of latent disease to active disease occurs in approximately $0.1 \%$ of people with LTBI per year. Consequently, the total number of latent cases was $3.1 \%$ (range $2.2 \%$ to $5.2 \%$ ) of the population in the years 2011-2015. With this information they were also able to make county level estimates. Information from individual states is not available in the NHANES data, but analysis of cases with active tuberculosis in individual states, such as Texas, can provide additional demographic and clinical information relevant to identifying individuals who likely have latent tuberculosis. In particular, adults with diabetes, HIV, a history of drug abuse, alcohol abuse, and adverse social circumstances, including incarceration and homelessness, are more likely to have latent tuberculosis.

The majority of patients with active tuberculosis in United States have had prior infection, i.e., have latent tuberculosis, and have had reactivation following changes in their health status. ${ }^{6}$ Consequently, patients with latent tuberculosis who are at increased risk for reactivation represent an important group of individuals who should undergo prophylactic treatment for latent tuberculosis when possible. The primary problem involves systematic identification of these patients and the supervision of a prolonged drug regimen that has the potential for toxicity. Most physicians with outpatient based clinical practices probably do not undertake the identification and treatment of these patients. Our review of the NHANES database identified very few participants who were on antituberculous medication. Vonnahme and colleagues also analyzed this database and calculated that $12.2 \%$ of all adults with a positive QuantiFERON test and a $13.5 \%$ of adults with a positive PPD test had received prior treatment for either latent or active tuberculosis. ${ }^{7}$
The primary responsibility of regional departments of health is to manage the treatment of patients with active infection and to do contact investigations. Therefore, most adults with latent tuberculosis will never have contact with a regional health department that specializes in the management of tuberculosis. Information available from NHANES and from summary information from the State Department of Health can help physicians and healthcare organizations identify patients who might benefit from screening for latent tuberculosis. Important groups include individuals who are foreign born, especially from Mexico, the Philippines, Vietnam, India, and China. ${ }^{8}$ In addition, individuals in high risk congregate settings, such as homeless shelters and correctional facilities, are at increased risk. These latter individuals often have personal characteristics which make screening and monitoring difficult. Other important groups include patients with immunosuppressive disorders, especially HIV, and patients on immunosuppressive medications.

Individuals in prisons and in jails reside in settings that can increase the risk of transmitting tuberculosis. The State of Texas has an organized program for the management of inmates with active infection and latent infections and who are possible contacts to individuals with active disease. ${ }^{9}$ The Texas Department of Criminal Justice has teams who track and monitor all tuberculosis screening and treatment activities, provide continuity of care, undertake contact investigation, and monitor completion of care for all inmates with active tuberculosis. For example, in 2017 the Texas Department of State Health Services reported that 94 individuals in correctional facilities had tuberculosis. ${ }^{4}$ In 2017, the Harris County Sheriff's Department (Houston) administered 21,752 tuberculin skin test, performed 2,867 chest x-rays, and identified 5 active cases. ${ }^{10}$ These efforts provide important benefits to individuals with active TB, limit transmission in congregate settings, and investigate possible contacts in and outside of the facility of inmates with active tuberculosis.

The CDC has developed a program for the management of immigrant and refugee health. This program requires a physical examination and investigation for communicable diseases, including 
tuberculosis, prior to entry into the United States. Analysis of this program indicates that a significant number of individuals complete treatment for pulmonary tuberculosis prior to entry into the United States and undergo a post-arrival evaluation. ${ }^{11}$ Statistics for this program published in November 2020 indicate that 4,225 persons completed treatment for pulmonary tuberculosis and 3005 underwent post-arrival evaluation. This evaluation diagnosed 22 additional cases of tuberculosis. Nearly sixty thousand individuals $(55,938)$ overseas had suspected tuberculosis but negative cultures, 37,089 completed post-arrival evaluation, and this resulted in the diagnosis of 597 cases. Latent tuberculosis was diagnosed in 30,574 individuals overseas, and 18,466 had post arrival evaluation that resulted in a diagnosis of 48 active cases. Treatment of latent tuberculosis was recommended for 21,714 persons following post-arrival evaluation, 14,977 individuals initiated this treatment, and 8,695 completed treatment. This program provides a framework for identifying and treating individuals with active tuberculosis and latent tuberculosis prior to entry into the US. The main difficulties likely involve the treatment of individuals who are asymptomatic and have only subtle abnormalities on chest $\mathrm{x}$-rays and the uptake and completion of treatment for latent tuberculosis.

The identification and treatment of individuals in the United States who do not fall in high risk categories present an important, difficult problem. These individuals likely receive primary healthcare from physicians who do not have much experience in the management of latent tuberculosis. One important approach would involve the identification and treatment of patients with chronic immunosuppression, such as HIV, malignancy, and organ transplantation. This responsibility should involve specialists in these disorders. The second approach would involve the dissemination of information to physicians in the healthcare system and to patients who use the healthcare system. This would require a short summary describing latent tuberculosis, tests needed to identify this clinical syndrome, and management options. Physicians could then screen patients based on their judgment, and patients could ask for screening if they think it is warranted.
Medical alerts can increase screening for infectious diseases, such as hepatitis C and HIV, but these alerts have definite problems and can cause alert fatigue. ${ }^{12,13}$ In addition, an important issue in the identification of adults with LTBI involves the interpretation of the test used. Ghassemieh et al. analyzed LTBI test agreement in the 2011-2012 NHANES database and concluded that there was only fair agreement between the PPD test and the QuantiFERON test and that this agreement depended on the cut points used for the various tests. ${ }^{14}$ They suggested that dual testing might be useful in some adults in certain circumstances. For example, an individual in a low risk group who had both a positive PPD and a positive QuantiFERON likely has latent tuberculosis. An individual in a high-risk group who has a negative PPD and a negative QuantiFERON likely does not have latent tuberculosis. Their analysis greatly increases the complexity of identifying patients with latent tuberculosis and operationally might require testing to be performed by specialized clinics. Our analysis of this database helps identify the characteristics of individuals with 2 negative tests and individuals with 2 positive tests. However, these two groups do not have unique features which help make identification easy.

The management of active tuberculosis and latent tuberculosis largely involves subspecialty clinics and public health departments. Although there has been a steady decline in the number of cases of tuberculosis in the United States, this remains an important public health problem. Multiple factors influence the frequency of primary infection and the development of active diseases, and these factors have the potential to change from year to year. Accurate reporting is essential to understand the current status in any particular region. The identification of individuals with latent tuberculosis requires a comprehensive program to evaluate at risk individuals. NHANES provides important information about the prevalence of latent tuberculosis and state reports clearly provide information about the number of active cases and their characteristics. Physicians and healthcare organizations need to understand and use this information. 
Article citation: Khan S, Ike C, Dennis J, Nugent K. The prevalence and characteristics of adults with latent tuberculous infection in the United States and the implications for healthcare in Texas. The Southwest Respiratory and Critical Care Chronicles 2021;9(39):53-62 From: School of Medicine (SK, Cl), Department of Public Health (JD), Department of Internal Medicine (KN), Texas Tech University Health Sciences Center, Lubbock, Texas

Submitted: $3 / 1 / 2021$

Accepted: 4/4/2021

Reviewer: Jacob Nichols MD

Conflicts of interest: none

This work is licensed under a Creative Commons

Attribution-ShareAlike 4.0 International License.

\section{REFERENCES}

1. Trends in Tuberculosis-United States, 2011. (2011). Centers for Disease Control and Prevention. https://www.cdc.gov/ $\mathrm{mmwr} / \mathrm{preview} / \mathrm{mmwrhtml} / \mathrm{mm} 6111 \mathrm{a} 2 . \mathrm{htm}$

2. NHANES 2011-2012: Tuberculosis Data Documentation, Codebook, and Frequencies. (2013). CDC.https://wwwn.cdc. gov/Nchs/Nhanes/2011-2012/TBX_G.htm

3. Mancuso JD, Diffenderfer JM, Ghassemieh BJ, et al. The prevalence of latent tuberculosis infection in the United States. Am J Respir Crit Care Med 2016;194(4):501-9.

4. Texas Department of State Health Services. TB Data and Statistics. Retrieved January 28, 2021, from https://www.dshs. texas.gov/idcu/disease/tb/statistics/

5. Haddad MB, Raz KM, Lash TL, et al. Simple estimates for local prevalence of latent tuberculosis infection,
United States, 2011-2015. Emerg Infect Dis 2018;24(10): 1930-1933.

6. Kiazyk S, Ball TB. Latent tuberculosis infection: An overview. Can Commun Dis Rep 2017;43(3-4):62-66.

7. Vonnahme LA, Haddad MB, Navin TR. Factoring prior treatment into tuberculosis infection prevalence estimates, United States, 2011-2012. Emerg Infect Dis 2019;25(10): 1949-1951.

8. Bibbins-Domingo K, Grossman DC, Curry SJ, et al. Screening for latent tuberculosis infection in adults: US Preventive Services Task Force Recommendation Statement. JAMA 2016;316(9):962-9.

9. DSHS Tuberculosis Standards for Texas Correctional and Detention Facilities. (n.d.). Texas Department of State Health Services.https:/www.dshs.texas.gov/IDCU/disease/tb/policies/ TBCorrectionalStandards.pdf

10. Harris County Sherrif's Department. (2017). Texas Department of State Health Services. https:/www.dshs.texas.gov/ IDCU/disease/tb/programs/jails/reports/17Harriscosherrifdept.pdf

11. Liu Y, Phares CR, Posey DL, et al. Tuberculosis among newly arrived immigrants and refugees in the United States. Ann Am Thorac Soc 2020;17(11):1401-1412.

12. Tapp H, Ludden T, Shade L, et al. Electronic medical record alert activation increase hepatitis $\mathrm{C}$ and HIV screening rates in primary care practices within a large healthcare system. Prev Med Rep 2020;17:101036. DOI: 10.1016/j.pmedr.2019. 101036.

13. Murphy DR, Reis B, Kadiyala H, etal. Electronic health recordbased messages to primary care providers: valuable information or just noise? Arch Intern Med 2012;172(3):283-5.

14. Ghassemieh BJ, Attia EF, Koelle DM, et al. Latent tuberculosis infection test agreement in the National Health and Nutrition Examination Survey. Am J Respir Crit Care Med 2016;194(4):493-500. 